\title{
Probability distribution for heat exchange in plastic deformation
}

\author{
W. Dednam $\odot,{ }^{1,2,3, *}$ M. J. Caturla $\odot,{ }^{2,3}$ A. E. Botha $\odot,{ }^{1}$ E. SanFabián $\odot,{ }^{2,4,5}$ J. A. Miralles, ${ }^{2,3}$ and E. Louis $\circledast^{2,3,4}$ \\ ${ }^{1}$ Department of Physics, Science Campus, University of South Africa, Florida Park, Johannesburg 1710, South Africa \\ ${ }^{2}$ Instituto Universitario de Materiales de Alicante (IUMA), Universidad de Alicante, San Vicente del Raspeig, E-03690 Alicante, Spain \\ ${ }^{3}$ Departamento de Física Aplicada, Universidad de Alicante, San Vicente del Raspeig, E-03690 Alicante, Spain \\ ${ }^{4}$ Unidad Asociada of the "Consejo Superior de Investigaciones Científicas,” Universidad de Alicante, San Vicente del Raspeig, \\ E-03690 Alicante, Spain \\ ${ }^{5}$ Departamento de Química Física, Universidad de Alicante, San Vicente del Raspeig, E-03690 Alicante, Spain
}

(Received 6 May 2021; accepted 20 August 2021; published 1 September 2021)

\begin{abstract}
Fluctuation theorems allow one to obtain equilibrium information from nonequilibrium experiments. The probability distribution function of the relevant magnitude measured along the irreversible nonequilibrium trajectories is an essential ingredient of fluctuation theorems. In small systems, where fluctuations can be larger than average values, probability distribution functions often deviate from being Gaussian, showing long tails, mostly exponential, and usually strongly asymmetric. Recently, the probability distribution function of the van Hove correlation function of the relevant magnitude was calculated, instead of that of the magnitude itself. The resulting probability distribution function is highly symmetric, obscuring the application of fluctuation theorems. Here, the discussion is illustrated with the help of results for the heat exchanged during plastic deformation of aluminum nanowires, obtained from molecular dynamics calculations. We find that the probability distribution function for the heat exchanged is centrally Gaussian, with asymmetric exponential tails further out. By calculating the symmetry function we show that this distribution is consistent with fluctuation theorems relating the differences between two equilibrium states to an infinite number of nonequilibrium paths connecting those two states.
\end{abstract}

DOI: 10.1103/PhysRevE.104.034101

Classical thermodynamics has facilitated a thorough understanding of the behavior of macroscopic systems. Its success, however, has not been restricted to the field of physics. Additionally, it has contributed to the development of chemistry and biology. Most systems of interest in these fields are in nonequilibrium steady states. Such states require one or various external agents to sustain them. These agents, or parameters, are the source of noise that in macroscopic systems leads to observed magnitudes that obey the central limit theorem (CLT), i.e., they take values distributed according to a Gaussian. The CLT is one of the most important theorems in statistics and is remarkably robust, applying to most natural and artificial systems with some degree of randomness, i.e., practically all systems. In the last 50 years the impressive development of experimental techniques has allowed us to work on and utilize very small systems well into the nanoscale. At these scales, fluctuations have amplitudes as large as average values, and thus it is unlikely that a classical approach will work.

Recently, several fluctuations theorems (FTs) have been developed [1], all having in common the possibility of calculating the difference in free energy of two equilibrium states in terms of the irreversible work done by the system over the infinite nonequilibrium trajectories connecting those states. The first serious attempt to open this door was the

\footnotetext{
*dednaw@unisa.ac.za
}

so-called Jarzynski equality proposed by Jarzynski in 1997 [2]. Two years later, Crooks [3] presented a generalization of the Jarzynski equality, while its usefulness was soon demonstrated experimentally [4,5]. Most fluctuation theorems can be written as [6]

$$
\frac{P(+F)}{P(-F)}=\exp \frac{F}{k_{B}} .
$$

$F$ has the dimensions of an entropy that may represent heat and/or work produced during a given time interval, and $P(+F)$ and $P(-F)$ correspond to the probability distribution functions (PDFs) of $F$ in the forward and reverse processes, respectively. In general, those probability distributions deviate from a Gaussian showing tails that most commonly are exponentials. Non-Gaussian tails have been observed in a wide variety of systems. The ratio of those two PDFs [left-hand side of Eq. (1)] can be referred to as the symmetry function,

$$
S(F)=\frac{P(+F)}{P(-F)} .
$$

The following works are only an illustration of the rich phenomenology already available: nonequilibrium fluctuations in a resistor [7], scalar turbulence [8] and turbulence [9-11], active gels [12], Brownian motion [13], DNA unzipping [14], disorder in the distribution of the interaction force between vortices in type II superconductors [15], nonGaussian tails in financial economics [16], plastic deformation of metallic nanowires $[17,18]$, and spontaneous heat exchange 
processes between quenched glasses and a heat bath [19]. Examples of power-law tails are sizes of the metastable intermediates in unzipped DNA exhibiting tails that follow a power law with a superexponential cutoff [14], Cauchy-Lorentz power-law distribution of out-of-plane jumps of carbon atoms in graphene [20], transitions from Gaussian to non-Gaussian velocity distribution functions in a vibrated granular bed [21], and fluctuations of the global velocity computed at various length scales during the intermittent mode-I propagation of a crack front [22]. In addition, several excellent reviews are available $[6,23,24]$.

In the following we shall concentrate on tails having an exponential character. Although such tails may be the result of rare events, they can dominate the physics of soft matter systems [13]. Specifically, we explore the appearance of these tails in nanoscale metallic systems at low temperature. Before proceeding it is mandatory to note that many authors, instead of calculating the PDF of the protocol parameter, say, for instance, the exchanged heat $Q$, computed the ensemble average of the so-called van Hove correlation function $[8,12,17]$,

$$
\Delta Q=Q(t)-Q(t+\tau),
$$

where $\tau$ is an arbitrary parameter, usually the time. It is worth noting that $\Delta Q$ is in most cases quite different from the standard correlation function [25],

$$
\delta Q=Q(t) Q(t+\tau)
$$

However, both $P(\Delta Q)$ and $P(\delta Q)$ are usually more symmetric and deviate less from a Gaussian than $P(Q)$, even though Eq. (3) [rather than Eq. (4)] is thought to be the more appropriate measure of correlation when the amplitude of the fluctuation is of the same order as that of the average itself [25].

To capture the asymmetry in $P(Q)$, and because deviations from a Gaussian can be of various kinds, we have tried to fit the numerical results for a PDF with the function

$$
P( \pm Q)=P(a)\left[\omega \exp \frac{(Q-a)^{2}}{-2 b^{2}}+(1-\omega) \exp \frac{(Q-a)}{\mp \lambda^{ \pm}}\right] .
$$

Parameter $a$ accounts for the fact that the most probable value of the protocol parameter is not always zero. Decaying of the Gaussian and the exponential terms is controlled by parameters $b$ and $\lambda^{ \pm}$, where the \pm sign accounts for the fact that tails may be different in forward $+(Q \geqslant 0)$ and reverse $(Q<0)$ processes $[1,10,14,19]$. In most cases we will divide the results for the PDF by its most probable value, and thus $P(Q=a)=1$.

In the following we shall illustrate these ideas with the help of numerical results for the heat exchanged during the plastic deformation of aluminum nanowires, obtained from an ensemble of molecular dynamics (MD) calculations. Details of the MD calculations can be found in Ref. [17] and a brief summary is included in the Appendix. As an aside, we note that the fluctuation theorems, as expressed through Jarzynski's equality, also apply to our simulations, which obey Nosé-Hoover dynamics [26-28].

Figure 1 shows the PDF of the heat exchanged during stretching of the aluminum wire (black symbols) and that of

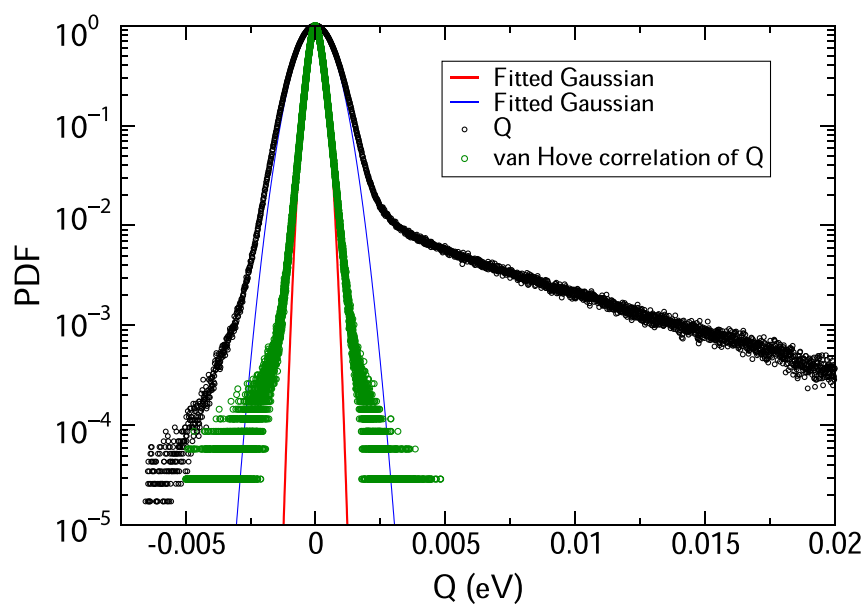

FIG. 1. Probability distribution function of heat exchanged in an aluminum wire stretched at constant speed (black line). The green curve corresponds to the van Hove correlation function $Q(t)-Q(t+$ $\tau$ ) (the data depicted in the figure were obtained with $\tau=10$ molecular dynamics steps-see the Appendix), where $\tau$ is an arbitrary parameter. The thin blue and red lines are Gaussians fitted only to the numerical data close to $Q=0$.

the van Hove correlation function of the heat data (green symbols). The two PDFs differ significantly. While the PDF of the heat is highly asymmetric showing a tail for $Q>0$ much longer than that for $Q<0$, the PDF of the correlation function is substantially more symmetric. Albeit most authors report asymmetric PDFs $[4,7,10,11,19,22]$, a significant amount of work has been done using the van Hove correlation function $[8,12,15,17]$ or even the standard correlation function $[13,20]$. In both cases symmetric PDFs are reported. There are also a few works presenting symmetric PDFs without specifying how they were obtained [16]. The present work shows results for both PDFs. Moreover, $P(+Q)$ and $P(-Q)$ would seem to correspond to different experimental realizations, but in practice they correspond in most cases to the same experiment and heat given or taken by the sample. For example, some authors measure $Q$ for folding and unfolding (zipping or unzipping) processes [5] but analyze the data as corresponding to different experiments. In the present case, the forward and reverse processes are very dissimilar and such an analysis of the data is thus not feasible.

Figure 2 depicts the fitting of $P( \pm Q)$ by means of Eq. (5). The most remarkable result is the large difference between the decaying length of the exponentials for negative and positive $Q$, almost an order of magnitude difference in Fig. 2(a) for the small system containing a few hundred atoms. The much longer exponential tail for $Q>0$ occurs because a large rearrangement of atoms due to a plastic deformation reduces the potential energy and converts it into kinetic energy that is absorbed by the heat reservoir. As the system size increases to a few thousand atoms, the exponential tails become relatively shorter, as one might expect. Such shortening can be seen clearly by comparing Figs. 2(a) and 2(b). The van Hove correlation function, Eq. (3), is necessarily symmetric because the rising kinetic energy is forced down every time the thermostat is applied. 
(a)

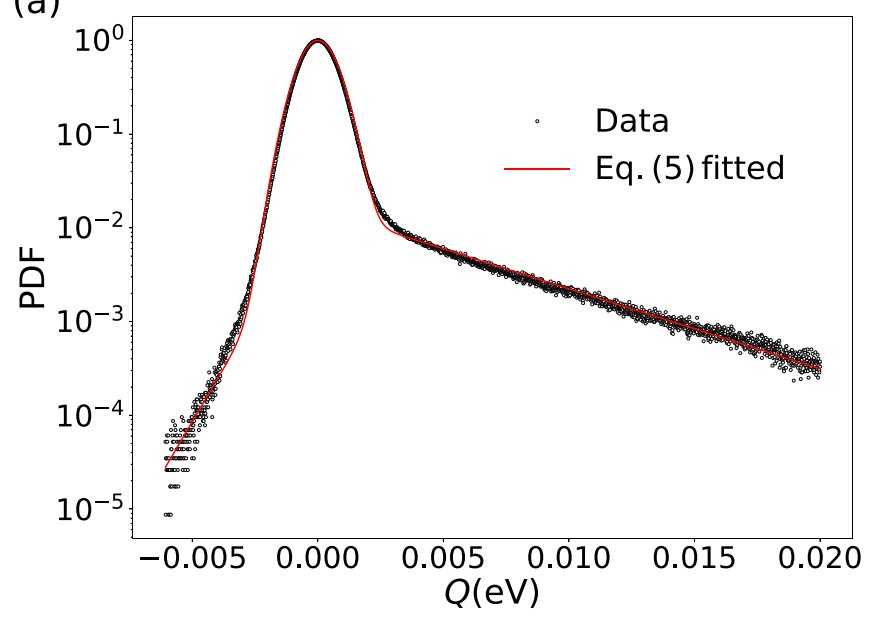

(b)

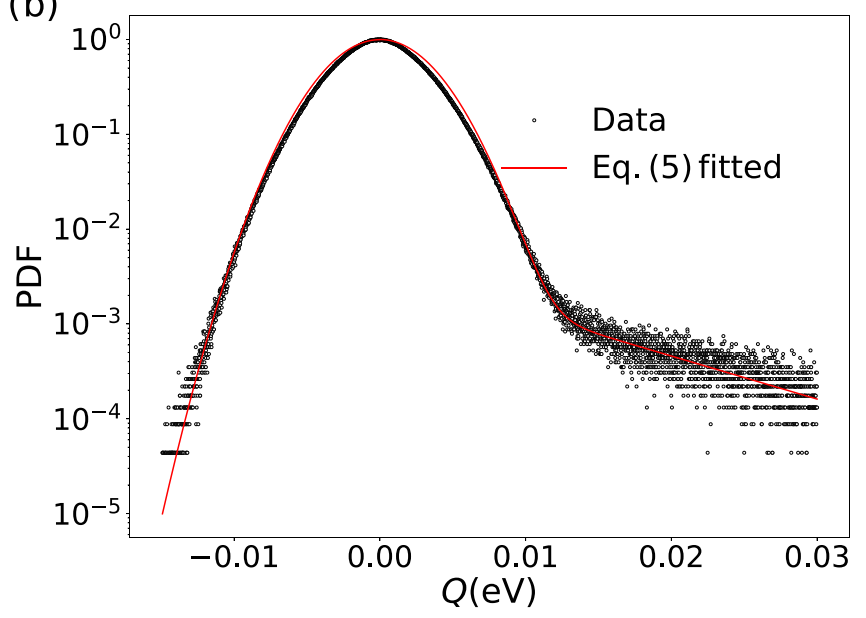

FIG. 2. (a) Fitting of $P(Q)$ in Fig. 1 with Eq. (5) and the following parameters: $\omega=0.984, a=0, b=0.000721, \lambda^{-}=0.000955$, and $\lambda^{+}=0.00508$. (b) Fitting of $P(Q)$ with Eq. (5) for a system containing several thousand atoms and using the following parameters: $\omega=0.996, a=0, b=0.00311, \lambda^{-}=5.95 \times 10^{-7}$, and $\lambda^{+}=$ 0.00956 .

In Fig. 3 we show the results corresponding to the symmetry function $S(Q)$, given by Eq. (2), for the same data set as in Fig. 2(a). As expected, $S(Q)=1$ for the region near $Q=0$, corresponding to the symmetric Gaussian part of the PDF. On the other hand, further out, in the spontaneous region, i.e., where the exponential tails show up, the symmetry function increases exponentially (blue fitted line), consistent with FTs having the form of Eq. (1).

In summary, we have performed a large ensemble of molecular dynamics simulations of the plastic deformation of aluminum nanowires at low temperature. Within the canonical ensemble, we computed the heat evolved during the stretching of the nanowires between applications of a thermostat every ten time steps. The resulting probability distribution of the heat evolved between the yield point and fracture of the nanowires can be fitted to a sum of a Gaussian function centered on zero, and two exponential tails which extend asymmetrically on either side of the Gaussian. Our calculations show that the van Hove correlations are distributed

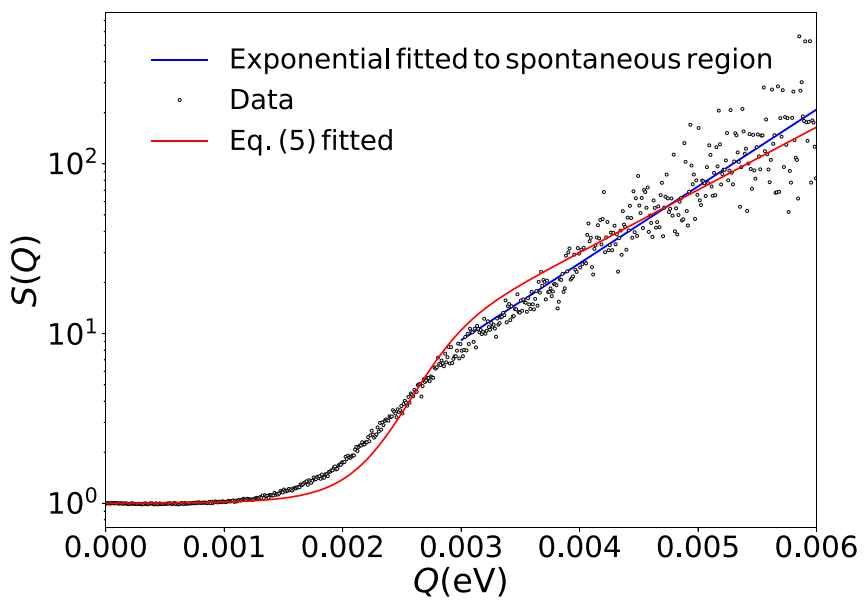

FIG. 3. Symmetry function $S(Q)$ as derived either directly from the numerical results of Fig. 2 or from the curve fitted to those data using Eq. (5). For comparison, the numerical data in only the tail region were fitted with an exponential function (blue line).

according to highly symmetric probability distribution functions. The PDFs of the undressed quantities, on the other hand, depend on the magnitudes of the quantities themselves, giving rise to the asymmetric PDF. Our simulation results dramatically support this conclusion.

The large deviation from Gaussian behavior appears to be indicative of strongly correlated disorder in the nanowires during plastic deformation processes that likely involve the nucleation of dislocations or the displacement of planes of atoms to accommodate the induced deformation. As such processes are inherently not stochastic, they do not follow a Gaussian distribution and are only observed at low temperatures when thermal fluctuations are very small. The fact that they are exponential may be related to an activation energy needed to generate a dislocation, or for planes of atoms to slip past each other.

Finally, in accordance with fluctuation theorems, near $Q=$ 0 , the symmetry function $S=1$. Barring a small amount of noise, for large $Q$, the symmetric function $S$ follows an exponential trend. These two limiting extremes are well matched where $S$ starts to deviate from one and reflect the great achievement of fluctuation theorems in relating differences between two equilibrium states to millions of nonequilibrium paths connecting those two states. Information on the nonequilibrium paths themselves can, in turn, be obtained experimentally or from ad hoc simulations.

This work was supported by "Ministerio de Ciencia e Innovación" of Spain (Grants No. FIS2015-64222-C22-P and No. PID2019-106114GB-I00), and the Spanish MEC (Grants No. MAT2005-07369-C03-01, No. NAN200409183-C10-08, and No. FIS2008-06743). M.J.C. acknowledges financial support from the Generalitat Valenciana through PROMETEO2017/139. J.A.M. acknowledges support from the Spanish Agencia Estatal de Investigación (PGC2018-095984-B-I00) and by the Generalitat Valenciana (PROMETEO/2019/071). The MD simulations in this paper 


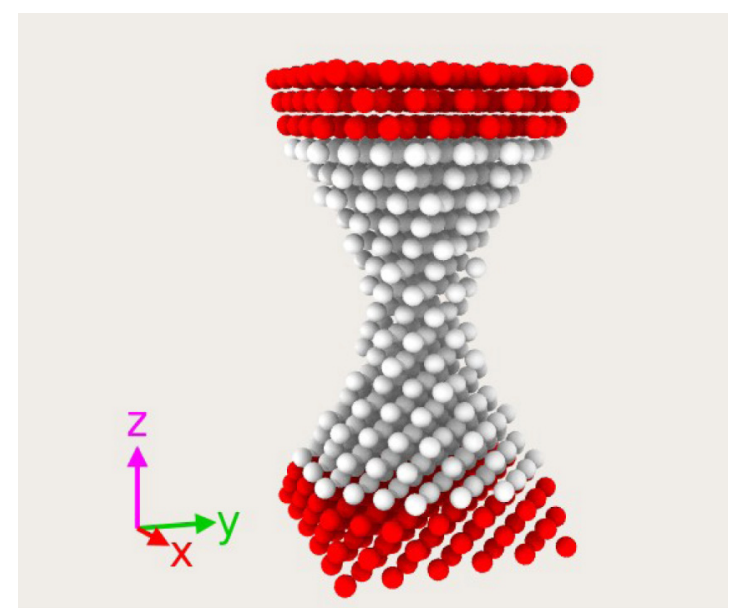

FIG. 4. A typical instance of a single-crystal aluminum nanowire consisting of 375 centrally located atoms (white) that are free to evolve dynamically and 178 atoms (red) on either end, which are constrained internally but are moved rigidly as a group in order to stretch the wire.

were performed on the high-performance computing facility of the University of South Africa.

\section{APPENDIX: MOLECULAR DYNAMICS CALCULATIONS}

Molecular dynamics (MD) simulations of single-crystal aluminum nanowires, containing $\sim 1000-5000$ atoms, were performed. Figure 4 shows a typical instance of a wire consisting of $N=731$ atoms. Notice that the cross section of the wire reduces towards its midsection to facilitate breaking. The large-scale atomic/molecular massively parallel simulator (LAMMPS) [29,30] was used for this purpose and the interatomic potential to describe the interactions between the atoms was taken from Ref. [31]. The nanowires were stretched along the [001] direction at a constant speed of $\sim 1.0 \mathrm{~m} \mathrm{~s}^{-1}$. Not all the atoms move freely and a few layers at the ends of the nanowire are frozen internally and moved rigidly as a group to stretch the nanowire (red atoms in Fig. 4). As initial conditions we took fixed atomic positions and the velocity at each "free" atom randomly distributed according to Maxwell distribution.

Before stretching, however, the energy of the wires was equilibrated for several tens of picoseconds within the microcanonical ensemble. Thereafter, simulations were done keeping the nanowire at a constant temperature of $0.5 \mathrm{~K}$, which amounts to assuming perfect thermal contact with a bath large enough to absorb all heat generated, an assumption that is justified in systems as small as those investigated here. This assumption was implemented in the simulations by thermostatting every ten MD steps all atom velocities using the Nosé-Hoover thermostat [26,27], known to be consistent

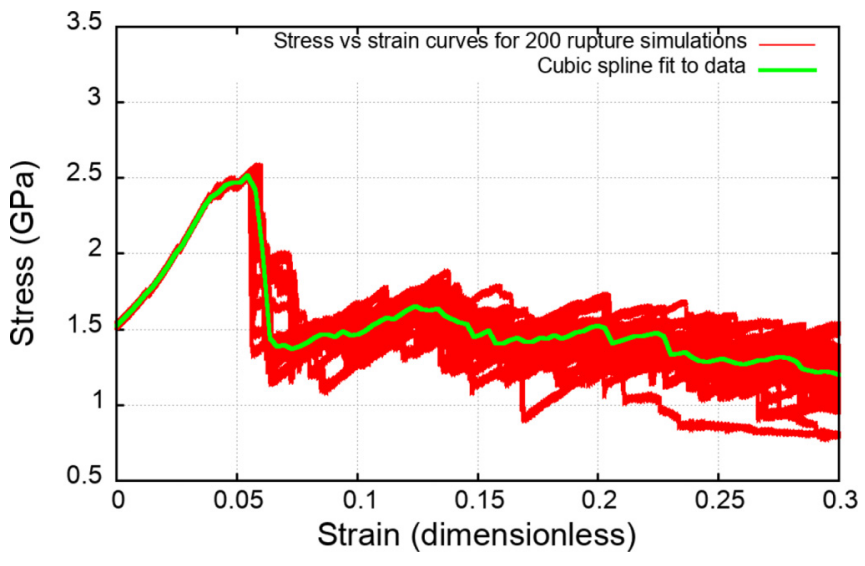

FIG. 5. Stress (GPa) vs strain curves (red lines) of an ensemble of rupture simulations and a cubic spline fit to the data (thick green line) showing the region of plastic deformations after the yield point, which occurs at a strain of roughly 0.05 .

with the canonical ensemble even in the presence of external forces [32,33].

In this work, the heat $(Q)$ data used to calculate the probability distribution functions (PDFs) shown in Figs. 1 and 2 of the main text are taken to be the difference between, on one hand, the kinetic energy just before thermostatting every ten MD time steps and, on the other hand, the target kinetic energy $3 N k_{\mathrm{B}} T / 2$, where $N$ is the number of "free" atoms, i.e., not in the frozen layers that are moved rigidly to stretch the wire, $k_{\mathrm{B}}$ is Boltzmann's constant, and $T=0.5 \mathrm{~K}$. Averaging is done over a large number of realizations (initial conditions) that is varied in order to check the convergence of the results. Subsequently, the heat exchanged during the large number of realizations is extracted from the simulations for those deformations in the stress-strain curve, calculated in LAMMPS based on the virial theorem, beyond the yield point and before breaking. In this region, the curves show steps composed by elastic regimes where the nanowire is stretched, followed by plastic deformation, where atomic rearrangements occur (see Fig. 5). Thus, the heat PDF was derived from the stress-strain curves as follows. First, it was checked that the yield point occurred at an extension that was similar for all realizations, near a strain of +0.05 and a stress of $\sim 2.5 \mathrm{GPa}$ in Fig. 5. Breaking in most cases occurred for extensions within a narrow range, but some samples broke either much earlier or much later. Then, only those realizations leading to extensions within that range were used (for strains between 0.075 and 0.3 in Fig. 5, or equivalently, all overlapping heat data between time step 300000 and 1200000 in the ensemble of simulations). For the nanowires and testing conditions described above, the number of points in the stress-strain curves used to produce the PDFs varied in the range $800-1000$, which gives a total of over 100000 points as in most cases the number of realizations was 200 .
[1] C. Bustamante, J. Liphardt, and F. Ritort, Phys. Today 58(7), 43 (2005), and references therein.

[2] C. Jarzynski, Phys. Rev. Lett. 78, 2690 (1997).
[3] G. E. Crooks, Phys. Rev. E 60, 2721 (1999).

[4] J. Liphardt, S. Dumond, S. B. Smith, I. Tinoco, Jr. and C. Bustamante, Science 296, 1832 (2002). 
[5] D. Collin, F. Ritort, C. Jarzynski, S. B. Smith, I. Tinoco, Jr. and C. Bustamante, Nature (London) 437, 231 (2005).

[6] F. Ritort, J. Phys.: Condens. Matter 18, 531 (2006).

[7] N. Garnier and S. Ciliberto, Phys. Rev. E 71, 060101(R) (2005).

[8] B. I. Shraiman and E. B. Siggia, Nature (London) 405, 639 (2000).

[9] S. T. Bramwell, P. C. W. Holdsworth, and J.-F. Pinton, Nature (London) 396, 552 (1998).

[10] Y. Li and C. Meneveau, J. Fluid Mech. 558, 233 (2006).

[11] Y. Li and C. Meneveau, Phys. Rev. Lett. 95, 164502 (2005).

[12] T. Toyota, D. A. Head, C. F. Schmidt, and D. Mizuno, Soft Matter 7, 3234 (2011).

[13] B. Wang, J. Kuo, S. C. Bae, and S. Granick, Nat. Mater. 11, 481 (2012).

[14] J. M. Huguet, N. Forns, and F. Ritort, Phys Rev. Lett. 103, 248106 (2009).

[15] J. A. Sánchez, G. Rumi, R. C. Maldonado, N. R. Cejas Bolecek, J. Puig, P. Pedrazzini, G. Nieva, M. I. Dolz, M. Konczykowski, C. J. van der Beek, A. B. Kolton, and Y. Fasano, Sci. Rep. 10, 19452 (2020).

[16] O. E. Barndorff-Nielsen and N. Shephard, J. R. Stat. Soc. B 63, 167 (2001).

[17] L. Pastor-Abia, M. J. Caturla, E. SanFabián, G. Chiappe, and E. Louis, Phys. Rev. B 78, 153410 (2008).

[18] L. Pastor-Abia, M. J. Caturla, E. SanFabián, G. Chiappe, and E. Louis, Phys. Rev. E 80, 030105(R) (2009).
[19] A. Crisanti and F. Ritort, Europhys. Lett. 66, 253 (2004).

[20] M. L. Ackerman, P. Kumar, M. Neek-Amal, P. M. Thibado, F. M. Peeters, and S. Singh, Phys. Rev. Lett. 117, 126801 (2016).

[21] Y. Murayama and M. Sano, J. Phys. Soc. Jpn. 67, 1826 (1998).

[22] K. T. Tallakstad, R. Toussaint, S. Santucci, and K. J. Måløy, Phys. Rev. Lett. 110, 145501 (2013)

[23] U. Seifert, Rep. Prog. Phys. 75, 126001 (2006).

[24] F. Ritort, Nonequilibrium fluctuations in small systems: From physics to biology, in Advances in Chemical Physics, Vol. 137, edited by S. A. Rice (Wiley Online Library, 2007), Chap. 2.

[25] L. van Hove, Phys. Rev. 95, 249 (1954).

[26] S. Nosé, Mol. Phys. 52, 255 (1984).

[27] W. G. Hoover, Phys. Rev. A 31, 1695 (1985).

[28] C. Jarzynski, Annu. Rev. Condens. Matter Phys. 2, 329 (2011).

[29] S. Plimpton, J. Comput. Phys. 117, 1 (1995).

[30] S. Plimpton, A. Thompson, S. Moore, A. Kohlmeyer, and R. Berger, Computer code LAMMPS, 2018, publicly available at http://lammps.sandia.gov.

[31] F. Ercolessi and J. B. Adams, Europhys. Lett. 26, 583 (1994).

[32] J. M. Haile, Molecular Dynamics Simulation (Wiley, New York, 1997).

[33] D. Frenkel and B. Smit, Understanding Molecular Dynamics Simulation (Academic, San Diego, 2002). 